\title{
Mean Diameter
}

National Cancer Institute

\section{Source}

National Cancer Institute. Mean Diameter. NCI Thesaurus. Code C120716.

The arithmetic mean of a range of values that describe the diameter of an entity. 